\title{
Theoretical interpretation of the X-ray properties of GRB 960720
}

\author{
F. Daigne and R. Mochkovitch \\ Institut d'Astrophysique de Paris, CNRS, 98 bis boulevard Arago, 75014 Paris, France
}

Received December 29, 1998; accepted March 9, 1999

\begin{abstract}
BeppoSAX observations of the single pulse burst GRB 960720 have allowed a detailed study of its $\mathrm{X}$-ray properties: pulse width in different energy bands, spectral evolution from 2 to $700 \mathrm{keV}$, etc. We show that the early $(0-5 \mathrm{~s}) \mathrm{X}$-ray emission and the gamma-rays are well explained by internal shocks in a relativistic wind while the late $(5-20 \mathrm{~s}) \mathrm{X}$-ray emission could come from the reverse shock generated in the wind when it interacts with the external medium. The results for a medium of uniform density are compared to the observations.
\end{abstract}

Key words: gamma-rays: bursts

\section{Introduction}

The gamma-ray emission from GRBs is probably produced by internal shocks in a relativistic wind whereas the afterglow (from X-rays to radio bands) is due to the external shock, i.e. the forward shock propagating in the ISM because of its interaction with the wind (Rees \& Mészáros 1994; Wijers et al. 1997). Simultaneously, a reverse shock propagates in the wind itself. We illustrate here the possible contribution of this reverse shock to a X-ray emission continuing immediately after the gamma-rays. Such emission has been observed in the first GRB detected by Beppo-SAX, GRB 960720. We use the detailed X-ray data made available by SAX for this burst (Piro et al. 1998) to make a comparison with our theoretical results.

\section{The internal shocks}

GRB 960720 has been observed both by BATSE and Beppo-SAX. It is a single-pulse burst, with a "FRED" profile. Its duration in the $50-700 \mathrm{keV}$ band is around $2-3 \mathrm{~s}$ but the X-ray emission lasts longer: Piro et al. (1998) show that the power-law between the width of the pulse and

Send offprint requests to: F. Daigne

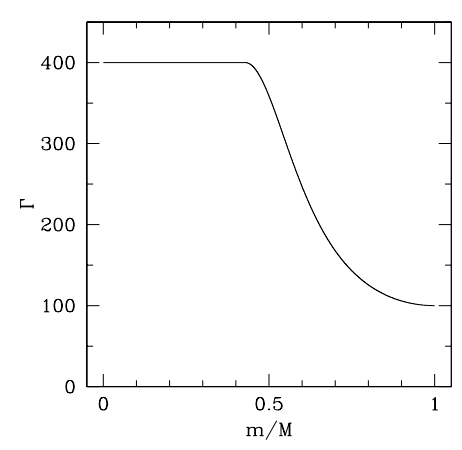

Fig. 1. Initial distribution of the Lorentz factor in the wind

the energy (already known in the gamma-ray range) is observed down to $2 \mathrm{keV}$. They find $W(E) \propto E^{-0.46}$.

We use a simple model to simulate internal shocks and build synthetic bursts: all pressure waves are neglected so that we consider only direct collisions between solid layers. In the shocked material, the magnetic field reaches equipartition values $(10-1000 \mathrm{G})$ and the Lorentz factor of the electrons is obtained from the dissipated energy per proton $\epsilon$ using the formula given by Bykov \& Mészáros (1996) who suppose that only a fraction $\zeta$ of the electrons is accelerated:

$\Gamma_{\mathrm{e}} \sim\left[\frac{\alpha_{\mathrm{M}}}{\zeta} \frac{\epsilon}{m_{\mathrm{e}} c^{2}}\right]^{\alpha}\left(\alpha_{\mathrm{M}} \sim 0.1 \rightarrow 1\right.$ and $\left.1 \leq \alpha \leq 1.5\right)$.

For $\zeta \sim 1$ the usual equipartition assumption yields values of $\Gamma_{\mathrm{e}}$ of a few hundreds: the gamma-ray emission is due to inverse Compton scattering on the synchrotron photons. Smaller values for the fraction of accelerated electrons $\left(\zeta<10^{-2}\right)$ lead to larger Lorenz factors $\left(\Gamma_{\mathrm{e}}\right.$ of a few thousands) so that the gamma-rays are directly produced by the synchrotron process, which is the assumption made here. Internal shocks have been shown to successfully reproduce the main temporal and spectral properties of GRBs (Daigne \& Mochkovitch 1998).

We model GRB 960720 with a wind emitted during $4 \mathrm{~s}$ and consisting in a slow and a rapid part of equal mass (see Fig. 1). Two internal shocks are generated and we sum both contributions to the emission to construct 

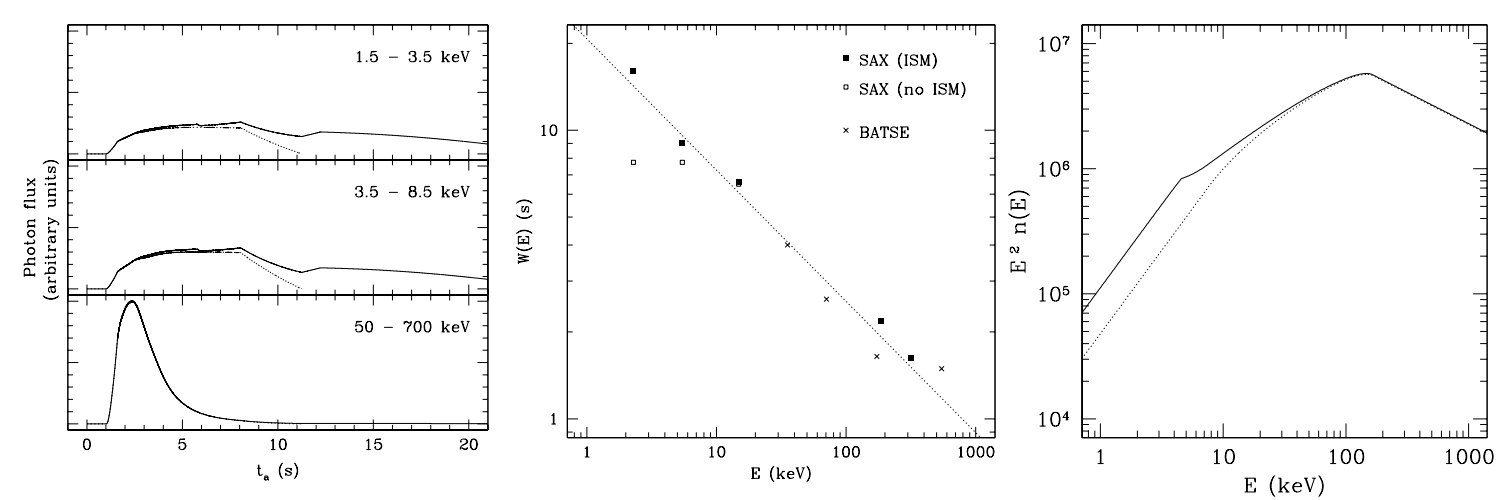

Fig. 2. Results obtained for a ratio $E_{52}^{\mathrm{inj}} / n=0.5$. For $E_{\gamma}=10^{51} / 4 \pi \mathrm{erg} / \mathrm{sr}$ and $f_{\gamma}=0.05$ it corresponds to $n=4 \mathrm{~cm}^{-3}$. Left panel: Profiles of the synthetic burst in the $\mathrm{X}$ - and gamma-ray bands. The full line takes into account the presence of the ISM whereas the dotted line only shows the contribution of the internal shocks. Middle panel: Pulse width as a function of energy. The dotted line corresponds to $W(E) \propto E^{-0.45}$. Right pannel: Burst spectrum. The full line takes into account the ISM and the dashed line does not

the synthetic burst. The profile in the SAX $50-700 \mathrm{keV}$ band looks very similar to GRB 960720 as can be seen in Fig. 2. However the X-ray emission does not last long enough so that the power-law relating $W(E)$ and $E$ is not reproduced in this spectral range.

\section{Effect of a medium of uniform density}

We now consider the effect of the ISM, whose density $n$ is supposed to be uniform. The external shock (forward shock) produces the afterglow. Simultaneously a reverse shock propagates into the wind. Its strength is comparable to that of the internal shocks, which are midly relativistic while the external shock is initially very strong and relativistic. We therefore adopt the same assumptions to compute the emission of the shocked material behind the reverse and the internal shocks.

It is possible to derive a critical ratio of the total energy injected over the density (see Sari \& Piran 1995):

$\left.\frac{E^{\text {inj }}}{n}\right|_{\text {crit }} \sim \frac{4 \pi}{3} m_{\mathrm{p}} c^{5} \bar{\Gamma}^{8} T^{3}$

for which the reverse shock will interfere with the internal shocks. Injecting the typical values used in our example (Lorentz factor $\bar{\Gamma} \sim 300$ and duration $T=4 \mathrm{~s}$ ) gives

$\left.\frac{E_{52}^{\text {inj }}}{n}\right|_{\text {crit }} \sim 0.071 \bar{\Gamma}_{300}^{8} T_{4}^{3}$,

where $E_{52}^{\mathrm{inj}}=\frac{E^{\mathrm{inj}}}{10^{52} / 4 \pi \mathrm{erg} / \mathrm{sr}}$. Assuming an efficiency $f_{\gamma}$ for the conversion of wind energy into gamma-rays by internal shocks allows to obtain $E^{\text {inj }}=\frac{E_{\gamma}}{f_{\gamma}}$ from the observed burst energy $E_{\gamma}$.

The total energy being fixed, if the density is smaller than the critical value, the reverse shock does not contribute in gamma-rays and only produces a delayed X-ray emission with an intensity which increases with $n$. If the density reaches or exceeds the critical value, the X-ray and the gamma-ray profiles become very affected.
We have represented in Fig. 2 the profiles for a ratio $E_{52}^{\text {inj }} / n=0.5\left(n \sim 0.15 n_{\text {crit }}\right)$. The gamma-ray profile is unchanged and the X-ray profiles are improved. The corresponding $E-W(E)$ diagram in Fig. 2 shows a well reproduced power-law over the complete energy range with an index -0.45 in agreement with the observations of GRB 960720. In the spectrum the contribution of the reverse shock to the late emission appears like a $\mathrm{X}$-ray plateau, which is observed (and is even more extended) in GRB 960720.

\section{Conclusions}

The reverse shock propagating into the wind following its interaction with the ISM has been shown to produce, for a sufficiently dense medium, a late X-ray emission. In the case of GRB 960720, whose gamma-ray properties are well explained by internal shocks, taking into account this additional contribution greatly improves the X-ray profiles (the power-law between the profile width and the energy is reproduced) and the spectrum.

It is now important to study the case of more complex environments. In hypernova models, the progenitor is a massive Wolf-Rayet star with a dense wind $(\dot{M} \sim$ $310^{-5} M_{\odot} / \mathrm{yr}$ and $v_{\infty} \sim 2000 \mathrm{~km} \mathrm{~s}^{-1}$ typically) leading to high densities $\left(n \sim \frac{\dot{M}}{4 \pi r^{2} m_{\mathrm{p}} v_{\infty}}\right)$ near the source. Our first calculations show that in this case even the gammaray profile could be strongly affected by the reverse shock, which may represent a potential problem.

\section{References}

Bykov A., Mészáros P., 1996, ApJ 461, L37

Daigne F., Mochkovitch R., 1998, MNRAS 296, 275

Piro L., et al., 1998, A\&A 329, 906

Rees M.J., Mészáros P., 1994, ApJ 430, L93

Sari R., Piran T., 1995, ApJ 455, L143

Wijers R.A.M.J., Rees M.J., Mészáros P., 1997, MNRAS 288, L51 